\title{
Use of different but overlapping determinants in a retrovirus receptor accounts for non-reciprocal interference between xenotropic and polytropic murine leukemia viruses Neal S Van Hoeven ${ }^{1,2,3}$ and A Dusty Miller*1
}

Address: ${ }^{1}$ Division of Human Biology, Fred Hutchinson Cancer Research Center, Seattle, Washington 98109, USA, ${ }^{2}$ Molecular and Cellular Biology Program, Fred Hutchinson Cancer Research Center, Seattle, Washington 98109, USA and ${ }^{3}$ Current address: Centers for Disease Control, Atlanta, Georgia 30333, USA

Email: Neal S Van Hoeven - nvanhoeven@cdc.gov; A Dusty Miller* - dmiller@fhcrc.org

* Corresponding author

Published: I5 December 2005

Retrovirology 2005, 2:76 doi:10.1186/1742-4690-2-76
Received: 13 September 2005

Accepted: 15 December 2005

This article is available from: http://www.retrovirology.com/content/2/I/76

(c) 2005 Van Hoeven and Miller; licensee BioMed Central Ltd.

This is an Open Access article distributed under the terms of the Creative Commons Attribution License (http://creativecommons.org/licenses/by/2.0), which permits unrestricted use, distribution, and reproduction in any medium, provided the original work is properly cited.

\begin{abstract}
Background: Retrovirus infection depends on binding of the retroviral envelope (Env) protein to specific cell-surface protein receptors. Interference, or superinfection resistance, is a frequent consequence of retroviral infection, and occurs when newly-synthesized Env binds to receptor proteins resulting in a block to entry by retroviruses that use the same receptors. Three groups of viruses demonstrate a non-reciprocal pattern of interference (NRI), which requires the existence of both a common receptor utilized by all viruses within the group, and a specific receptor that is used by a subset of viruses. In the case of amphotropic and IOAI murine leukemia viruses (MLV), the common and specific receptors are the products of two related genes. In the case of avian sarcoma and leukosis virus types $\mathrm{B}, \mathrm{D}$, and $\mathrm{E}$, the two receptors are distinct protein products of a single gene. NRI also occurs between xenotropic and polytropic MLV. The common receptor, Xprl, has been identified, but a specific receptor has yet to be described.

Results: Using chimeric receptor proteins and interference studies, we have identified a region of $\mathrm{Xprl}$ that is uniquely utilized by xenotropic MLV and show that this receptor domain is required for non-reciprocal interference.

Conclusion: We propose a novel pattern of receptor usage by xenotropic and polytropic MLV to explain the NRI observed between these viruses. We propose that the specific and common receptor determinants for xenotropic and polytropic viruses are simultaneously present in discreet domains of a single Xprl protein.
\end{abstract}

\section{Background}

Retroviral infection of a host cell is initiated by interaction of the retroviral Env protein surface (SU) subunit with a specific host cell receptor. This interaction triggers conformational changes within the Env protein that bring the virus and host cell membranes in close proximity, result- ing in fusion and delivery of the viral capsids into the host cell cytoplasm (reviewed in $[1,2]$ ). In addition to promoting virus entry, the intracellular interaction of a viral Env and its cognate receptor can limit subsequent infection by subsequent viruses that bind the same receptor. This phenotype is referred to as interference or superinfection 

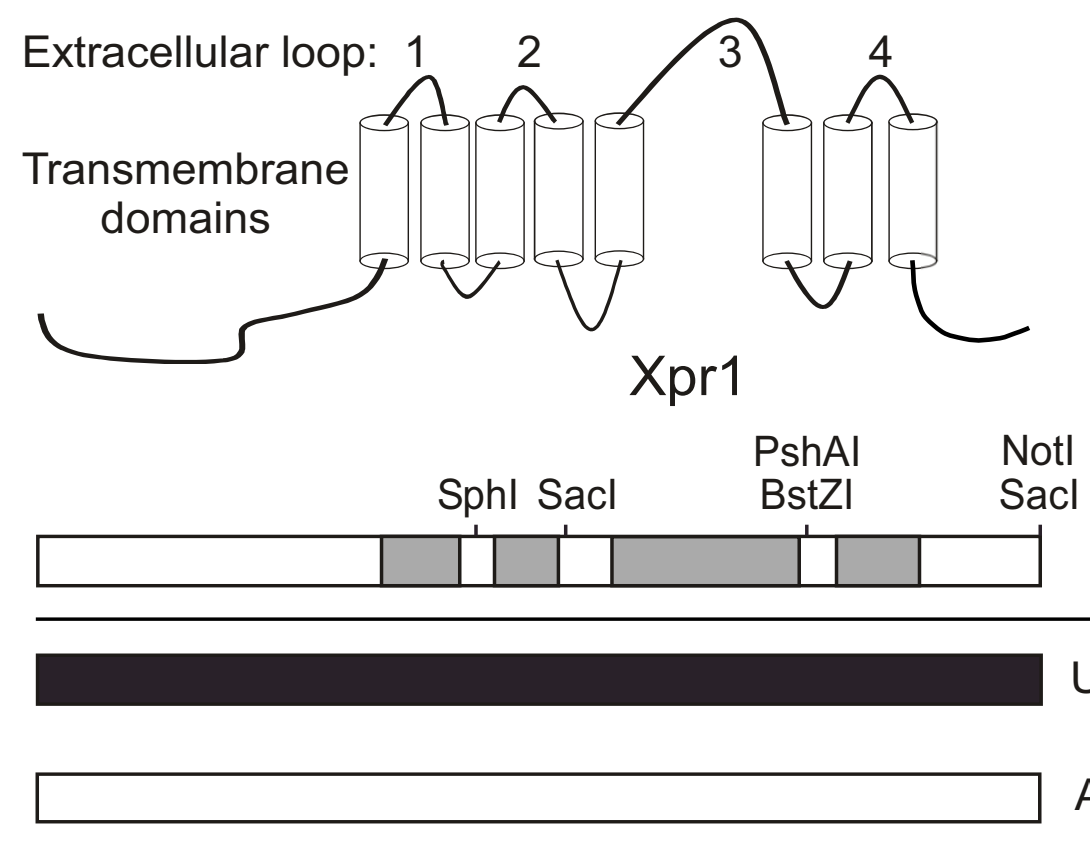

\section{UUUU $2 \times 10^{6} 7 \times 10^{4}$}

$A A A A<10$

20

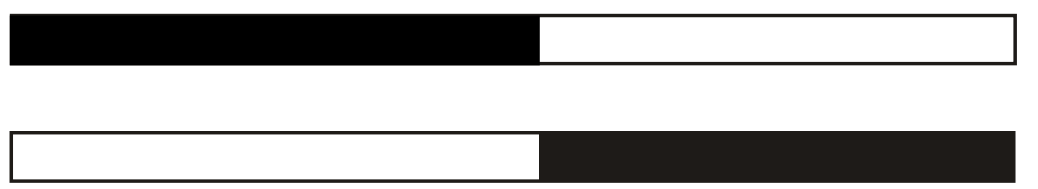

UUAA $\quad<10$

80

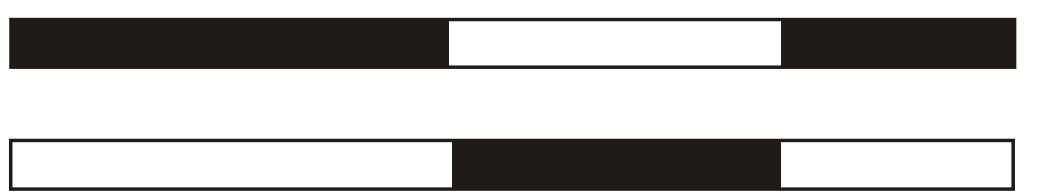

AAUU $8 \times 10^{5} 1 \times 10^{4}$

UAAU $3 \times 10^{4} 4 \times 10^{3}$

AUUA $1 \times 10^{6} \quad 10$

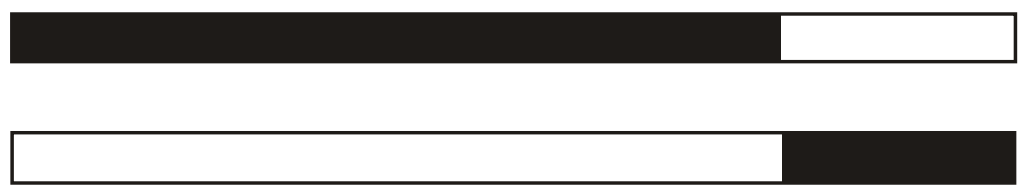

UUUA $5 \times 10^{5}<10$

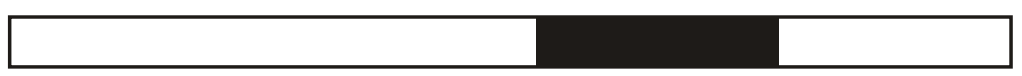

AAAU $4 \times 10^{5}$

$2 \times 10^{4}$

No added receptor

AAUA $3 \times 10^{6}$

30

$$
<10
$$

20

\section{Figure I}

Analysis of human/hamster $\mathrm{Xprl}$ chimeras for receptor function. The predicted transmembrane domain structure of $\mathrm{Xprl}$ is shown at top and a corresponding block diagram is shown just below with the extracellular loops (ECL) shown in grey. A series of chimeras were constructed by exchange of the indicated fragments of $\mathrm{hXprl}$ and haXprl. Restriction enzyme sites used in construction of the $\mathrm{Xprl}$ chimeras are shown above the block diagram. Chimeric receptors were subcloned into a retroviral expression vector and were transfected into $\mathrm{CHO}$ cells. The cells were then grown in medium containing G4I8 to select for expression of the Neo gene also carried by the expression plasmid. Cells were then exposed to LAPSN vectors bearing either the AKR6 or the IE Env and the apparent titers of the vectors were determined. Results are means of at least two independent experiments with triplicate determinations in each experiment. 
resistance because it prevents reinfection of a cell by the same virus strain, and has been used to classify viruses that utilize common cell surface receptors. Currently, mammalian retroviruses are divided into at least 10 different interference groups $[3,4]$. Within these groups, several retroviruses show a non-reciprocal interference pattern (NRI), where infection by one virus will block infection by a second virus, but infection by the second virus only slightly inhibits infection by the first virus.

As the receptors for different retroviruses have been identified, it has become clear that NRI occurs in cases where related viruses within an interference group utilize a partially overlapping set of receptors for entry. In the case of amphotropic and 10A1 MLV [5] these receptors are Pit1 (Slc20a1) and Pit2 (Slc20a2), the products of two different genes with similar sequence and function. The phosphate transporter Pit2 serves as the receptor for both amphotropic MLV [6,7] and 10A1 [8]. However, 10A1 also binds to the closely related phosphate transporter Pit1, the receptor for gibbon ape leukemia virus (GALV) [9] and feline leukemia virus subtype B (FeLV-B) [10]. Because the amphotropic Env cannot bind to Pit1, it cannot block 10A1 infection of cells that express both receptors, while the 10A1 Env can block amphotropic MLV infection [8].

NRI also occurs among avian sarcoma and leukosis viruses (ASLV) types B, D, and E. Viruses of types B and D can interfere with each other as well as type E viruses, whereas ASLV-E can interfere with itself, but not with types $\mathrm{B}$ or $\mathrm{D}$. This group of viruses have all been shown to utilize a common receptor, CAR1 $[11,12]$. Immunoprecipitation studies with different viral Env proteins have shown that this protein, encoded by the $t v-b$ locus in chickens, produces two distinct protein products that differ in their disulfide bond pattern. One form, designated the type 1 receptor, can interact with ASLV-B and ASLV-E, whereas an additional form, the type 2 receptor, is specific for ASLV-B [13].

Another set of retroviruses that show NRI are xenotropic and polytropic MLV (X-MLV and P-MLV, respectively). Studies in cells derived from mink and the wild mouse Mus dunni demonstrated NRI between X-MLV and P-MLV $[4,14]$, implying the existence of a common receptor. In both cases, initial infection of cells with X-MLV strains resulted in complete resistance to subsequent infection by P-MLV isolates. However, initial infection of cells with PMLV strains did not block infection by X-MLV, although the X-MLV titers observed were decreased [4,14]. The hypothesis that these viruses share a common receptor was confirmed by the identification of a single cDNA from humans [15,16] and mice [17] that could mediate infection of both viruses when expressed in resistant cells.
However, the identification of a single cell surface receptor is inconsistent with the interference patterns observed between these two viruses. Previously established mechanisms of NRI would suggest the existence of a specific XMLV receptor that cannot be utilized by P-MLV. Screening of cDNA libraries by three groups independently failed to identify additional genes encoding a xenotropic specific receptor. Furthermore, genetic studies in mice have mapped susceptibility loci for xenotropic and polytropic viruses to the same region of mouse chromosome 1 , and it is currently believed that these studies have identified alleles of the same gene $[18,19]$. These studies collectively argue against the existence of a separate locus encoding an $\mathrm{X}-\mathrm{MLV}$ specific receptor, and suggest that the specific and the common receptor are encoded by the same gene.

The common receptor, designated Xpr1, is a multiple-pass transmembrane protein of unknown function, although the gene displays a high homology to the Saccharomyces cerevisiae Syg1 gene. In yeast, Syg1 is involved in regulation of G-protein mediated signaling [20]. Current topology models predict that the receptor contains four extracellular loops (ECL), and intracellular amino and carboxy termini (Figure 1). Studies subsequent to the identification of the receptor have found residues within the putative third and fourth ECL, at amino acid positions 500 and 582 of the NIH Swiss mouse Xpr1 protein (mXpr1), that are critical for X-MLV receptor function [21]. Due to the ability of P-MLV isolates to utilize mXpr1, a similar set of residues required for P-MLV function were not identified. Our initial studies have focused on examining the determinants for both X-MLV and P-MLV in the same receptor. Making use of chimeras between the functional human and the nonfunctional hamster Xpr1 orthologs, we have identified regions of human Xpr1 that are sufficient to generate functional receptors for xenotropic and polytropic viruses. These studies suggest that two entry determinants are present on Xpr1. One determinant in the putative fourth ECL can be utilized by X-MLV and P-MLV, while a second determinant present in the third ECL can only be used by X-MLV. These results and additional interference studies support a novel model to explain NRI between these two virus types and have identified the xenotropic-specific receptor determinant as a particular domain of Xpr1.

\section{Results \\ Role of the putative third and fourth ECL of Xprl in xenotropic and polytropic virus entry}

To identify regions of human Xpr1 (hXpr1) that are required for xenotropic and polytropic virus receptor function, chimeric receptors combining coding sequences from hXpr1 and from the non-functional hamster receptor (haXpr1) were made and tested for receptor function following expression in Chinese hamster ovary (CHO) 

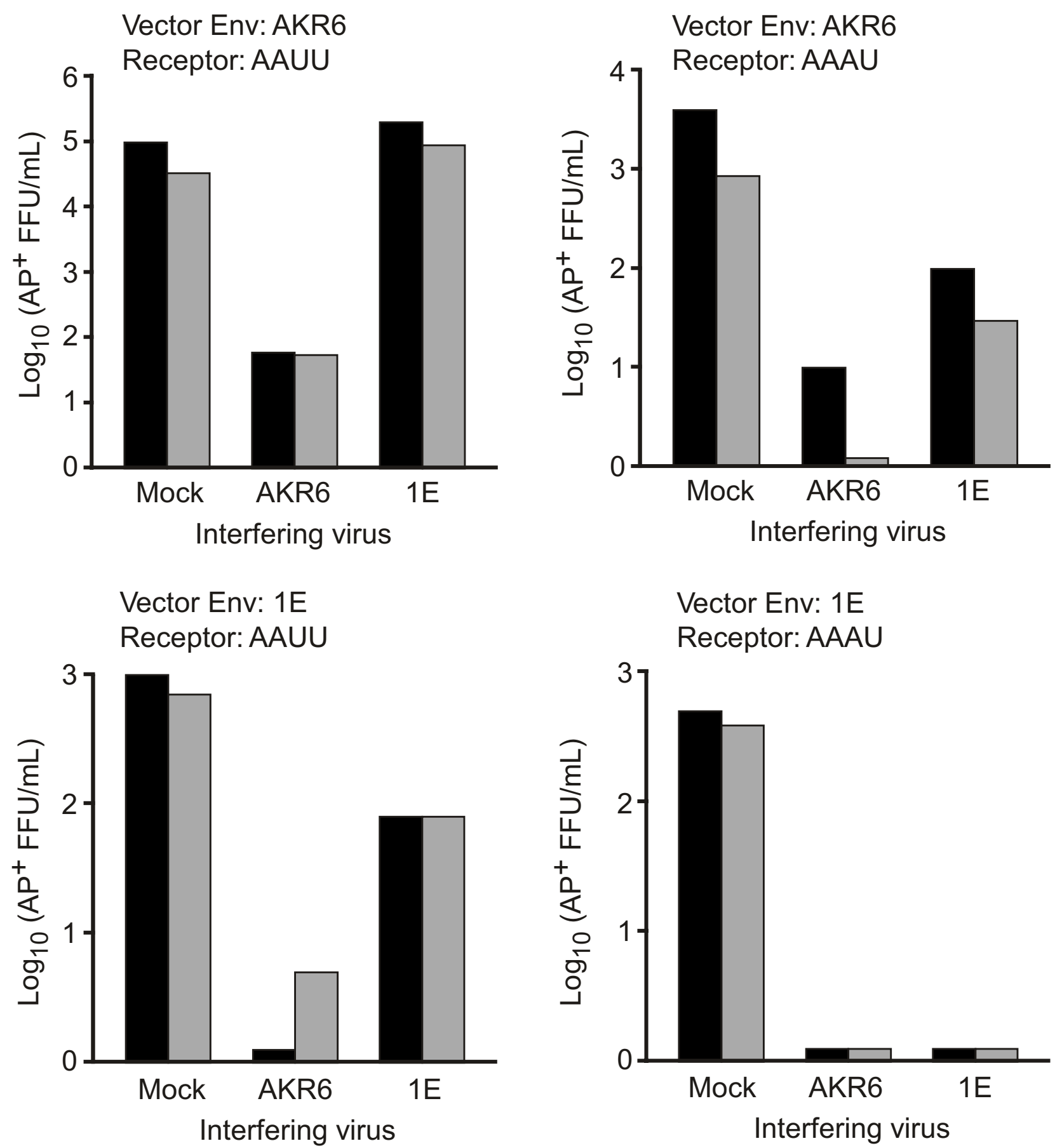

\section{Figure 2}

Analysis of AKR6 and IE virus interference in CHO cells expressing the AAUU and AAAU chimeric receptors. $\mathrm{CHO}$ cells transduced by retroviral vectors expressing the chimeric receptors AAUU or AAAU were infected with AKR6 or IE viruses by maintenance of the cells in virus-containing medium or in standard medium (mock infected) for six weeks. After infection the cells were seeded into 6-cm-diameter dishes, were exposed to vectors bearing the indicated Env, and vector titers were determined. Data from two independent infection/vector-titer-measurement experiments, one represented by grey boxes and the other by black boxes, are shown. Titer measurements in each experiment were performed in triplicate. 
cells (Figure 1). Chimeric receptors were named based on the order of human (U) and hamster (A) sequences that include the putative extracellular domains of the receptor. Because CHO cells can be infected by some X-MLV strains, we used the Env from an X-MLV strain (AKR6) that was unable to mediate transduction of $\mathrm{CHO}$ cells even when haXpr1 was overexpressed in the cells (Figure 1, construct AAAA). We also tested the Env from a P-MLV strain (1E) of Friend mink cell focus-forming virus (FrMCF) that mediates only a low rate of transduction of $\mathrm{CHO}$ cells overexpressing haXpr1 (Figure 1, construct AAAA). Both Env proteins could mediate relatively efficient transduction of CHO cells expressing hXpr1 (Figure 1, construct UUUU).

CHO cells expressing the Xpr1 chimeras were exposed to xenotropic [LAPSN(AKR6)] or polytropic [LAPSN(1E)] vectors and vector titers were determined (Figure 1). Cells expressing the UUAA chimera were poorly transduced by LAPSN(AKR6) or LAPSN(1E). Conversely, cells expressing the AAUU chimera were transduced at levels only slightly lower than those observed for hXpr1, indicating that the third and fourth loops of hXpr1 are important for both xenotropic and polytropic virus receptor function. Additional analysis of the determinants in this region shows that either the third or the fourth ECL is sufficient for xenotropic virus entry, but that only the fourth ECL can mediate polytropic virus entry. In particular, the AKR6 xenotropic vector could efficiently transduce cells expressing the AAAU or the AAUA chimeras, while the 1E polytropic vector could infect cells expressing the AAAU chimera but not the AAUA chimera.

\section{Xenotropic and polytropic Env show reciprocal interference on some chimeric receptors}

In previous interference studies, infection with a xenotropic virus blocks subsequent infection by viruses bearing either xenotropic or polytropic Env. In contrast, expression of a polytropic Env blocks subsequent infection by other polytropic viruses, but only slightly inhibits xenotropic infection $[4,14]$. Using our chimeric Xpr1 proteins, we examined the requirement for different regions of Xpr1 in interference between AKR6 and 1E pseudotype vectors.

To establish $\mathrm{CHO}$ cell lines expressing both a chimeric Xpr1 receptor and a retroviral Env, $\mathrm{CHO}$ cells were transduced with retroviral vectors expressing the chimeric receptors and were then maintained in medium containing replication-competent AKR6 or 1E virus for a period of 6 weeks, as described in Materials and Methods. Cells expressing Xpr1 chimeras and viral Env proteins were challenged with LAPSN(AKR6) or LAPSN(1E) vectors. The level of interference was determined by comparing the titers of LAPSN(AKR6) and LASPN(1E) vectors on mock infected cells versus that on cells infected with a replication competent virus. In CHO cells expressing the AAUU chimera we observed a non-reciprocal pattern of interference between AKR6 and 1E viruses (Figure 2, left panels) similar to that reported previously. Specifically, $\mathrm{CHO} /$ AAUU cells infected with AKR6 virus were refractory to transduction by both LAPSN(AKR6) and LAPSN(1E), while $\mathrm{CHO} / \mathrm{AAUU}$ cells infected with $1 \mathrm{E}$ virus were fully susceptible to transduction by LAPSN(AKR6) and were somewhat resistant to transduction by LAPSN(1E). The weak resistance of the 1E-infected CHO/LAAUUSN cells to transduction by LAPSN(1E) is somewhat surprising given that significant levels of interference have previously been described with this Env [4]. The titer we observed was only 10 fold lower than that observed in mock infected CHO/LAAUUSN cells, but was reproduced in multiple independent experiments. Taken together, these results demonstrate NRI for xenotropic and polytropic viruses in $\mathrm{CHO}$ cells expressing the AAUU chimeric receptor, similar to that observed previously for xenotropic and polytropic viruses.

The interference patterns on CHO/AAAU cells were markedly different from those described for CHO/AAUU cells. The AAAU receptor contains only a single entry determinant that can be utilized by both AKR6 and 1E pseudotyped viruses. In cells expressing this receptor, transduction by the LAPSN(AKR6) or LAPSN(1E) vectors was blocked by the presence of either AKR6 or 1E Env (Figure 2, right panels), thus showing a pattern of reciprocal interference. Although transduction by LAPSN(AKR6) was not completely blocked by $1 \mathrm{E}$ Env, a similar degree of interference was observed in two independent experiments, and the observed differences in titer were found to be statistically significant in both cases by using the Student's t-test $(p<0.05)$.

In summary, these experiments demonstrate a non-reciprocal interference pattern between AKR6 and polytropic viruses on the AAUU chimera, and a reciprocal pattern of interference in the AAAU chimera, which contains only the putative fourth ECL of human Xpr1. These results support the hypothesis that xenotropic virus can utilize either the third or fourth ECL of hXpr1 for cell entry, but that polytropic virus can only use the fourth ECL. When the third ECL is replaced with the non-functional loop from haXpr1, both viruses can only use the fourth ECL for entry and therefore show reciprocal interference.

\section{SU domains of AKR6 and IE Env show high sequence similarity to prototypical xenotropic and polytropic Env SU domains}

To characterize the interaction of AKR6 and 1E Env proteins with Xpr1 in more detail, we isolated and cloned the receptor-binding surface (SU) subunits from both pro- 


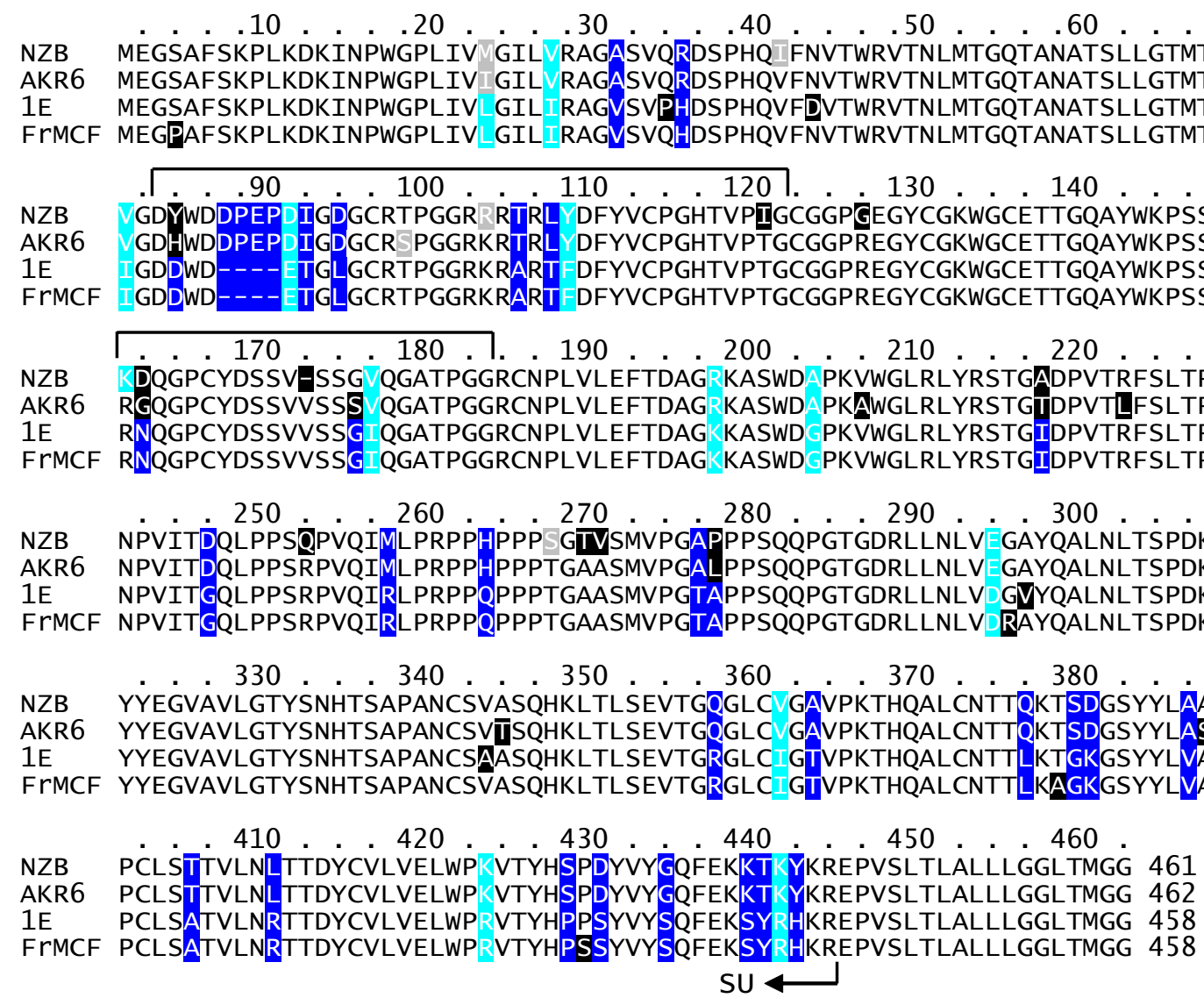

Figure 3

Amino acid sequence comparison of the Env SU domains of AKR6 X-MLV, IE P-MLV, and prototypic X-MLV and P-MLV. Amino acid alignment of the Env SU domains of NZB X-MLV [GenBank:K02730], AKR6 X-MLV [GenBank:DQ199948], IE P-MLV [GenBank:DQ199949], and FrMCF P-MLV [GenBank:X01679]. Sequences are shown starting with the initiator methionine and include endoplasmic reticulum signal sequences of unknown lengths. Variable regions $A$ and $B$, believed to be responsible for receptor recognition [45], are indicated by brackets. Non-conservative amino acids differences are indicated by black boxes and conservative changes are indicated by grey boxes. Blue boxes indicate amino acids that are identical among the P-MLVs but dissimilar from one or more of those of the X-MLVs, identical among the X-MLVs but dissimilar from one or more of those of the P-MLVs, or both. Cyan boxes indicate amino acids that are identical among the PMLVs and similar to those of the X-MLVs, identical among the X-MLVs and similar to those of the P-MLVs, or both.

teins. The sequence of the SU region of each Env protein was determined by sequencing a PCR fragment isolated from Hirt DNA extracted from virus-infected dunni cells. Amino acid sequence alignments of AKR6 and 1E SU regions and the those of the prototypic NZB X-MLV $[22,23]$ and FrMCF P-MLV [24] strains shows that the 1E sequence is most like that of the FrMCF virus and the AKR6 sequence is most like that of the NZB sequence (Figure 3). For example, the $1 \mathrm{E}$ Env sequence contains a four residue deletion relative to NZB and AKR6 xenotropic Env proteins that is also present in the FrMCF polytropic Env. Additional sequence differences between the Env proteins, many of which occur in two variable regions, are likely to account for differences in host range observed between these viruses.

A full-length env gene containing the cloned AKR6 SU sequence and the transmembrane (TM) subunit sequence from NZB X-MLV was constructed and was transfected into LGPS/LAPSN cells to generate LAPSN(AKRGenv) virus. The titer of this virus on dunni cells was $3 \times 10^{4} \mathrm{AP}^{+}$ FFU/ml. To verify the identity of the cloned AKR6 Env, we measured the titer of the LAPSN(AKRGenv) vector on dunni cells previously infected with replication competent AKR6 or 1E viruses (Figure 4A). LAPSN(AKR6env) transduction of dunni/AKR6 cells was almost completely 

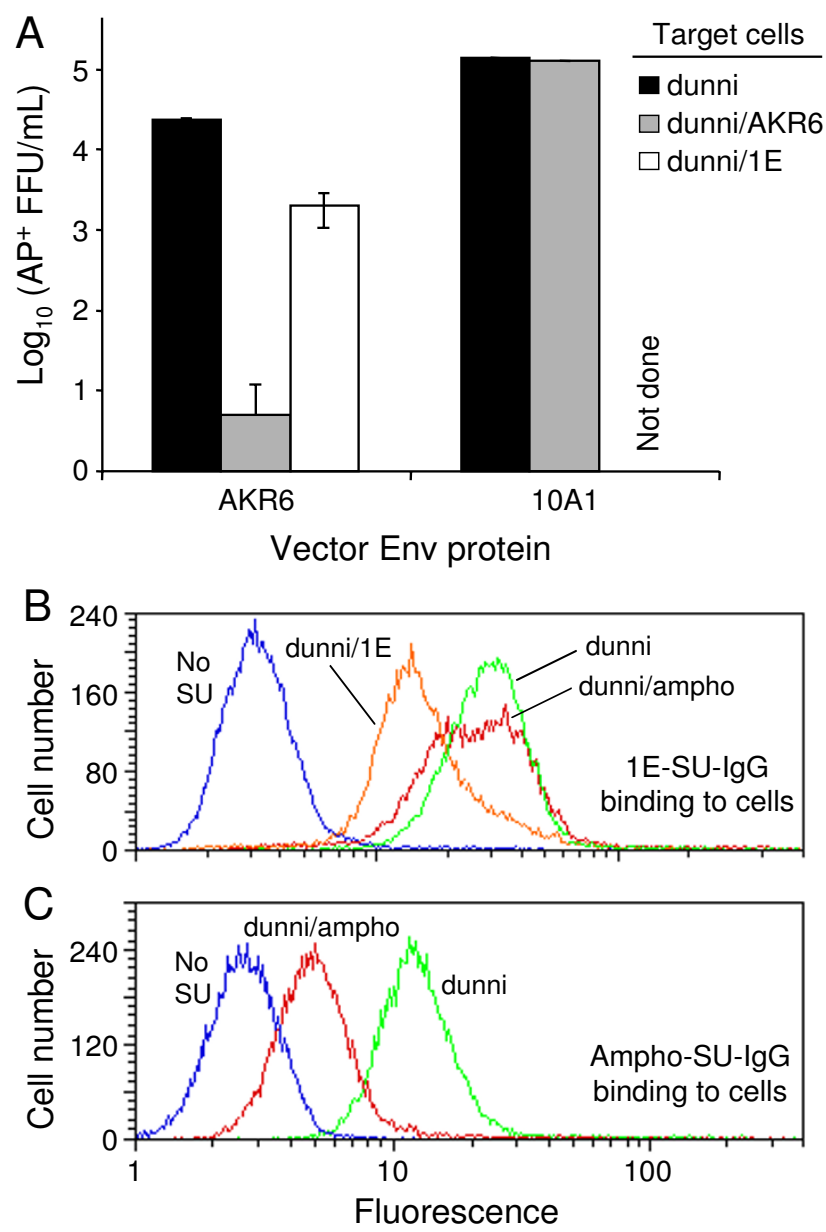

\section{Figure 4}

Binding and interference properties of cloned AKR6 SU and IE SU. (A) LAPSN(AKR6env) and

LAPSN(IOA I env) vector titers were measured on dunni cells and dunni cells infected with replication-competent AKR6 or $I E$ viruses. Data shown are means \pm SD of at least two independent experiments with duplicate determinations in each experiment. (B) Binding of IE-SU-IgG to dunni cells and to dunni cells infected with replication-competent viruses. (C) Binding of Ampho-SU-lgG to dunni cells infected with 4070A amphotropic virus. Data in (B) and $(C)$ are from a representative experiment and show data from $\sim \mid 8,000$ live cells (cells that exclude propidium iodide) per histogram.

blocked $\left(<10 \mathrm{AP}^{+} \mathrm{FFU} / \mathrm{ml}\right)$. In contrast, the titer of this vector on dunni/1E cells was reduced by only about 10fold. As a control, the titer of LAPSN(10A1) vector on dunni and dunni/AKR6 cells was also measured. The 10A1 Env utilizes Pit1 and/or Pit 2 for entry, and so should not be affected by the presence of AKR6 xenotropic Env in the cells. As expected, the LAPSN(10A1) titers were equivalent on these cell lines (Figure 4A). The block to LAPSN(AKR6env) transduction in cells chronically infected with AKR6 suggests that the cloned sequence encodes a protein that binds the same receptor as biological isolates of AKR6. Furthermore, the infection patterns observed on dunni/AKR6 and dunni/1E cells are consistent with the NRI previously observed for X-MLV and PMLV.

A full-length env gene containing the cloned 1E SU sequence and the transmembrane (TM) subunit sequence from NZB X-MLV was constructed and was transfected into LGPS/LAPSN cells, but vector production from these cells was not detected. Examination of multiple 1E-SU PCR clones isolated from various Hirt preparations of $1 \mathrm{E}$ virus DNA indicated that the $1 \mathrm{E}-\mathrm{SU}$ clone we used to construct the Env expression vector does not contain inactivating mutations. Attempts to clone the remaining TM sequence from 1E Env by PCR using primers to conserved regions of Env were unsuccessful, suggesting that $1 \mathrm{E}$ may have unique sequences present in the TM domain that are required for proper Env function.

To verify that the cloned $1 \mathrm{E}$ SU sequence had the properties of a polytropic virus SU domain, we generated a human IgG tagged version of 1E-SU (1E-SU-IgG). Following production of the protein by transient transfection and purification by FPLC, we examined the binding of $1 \mathrm{E}-$ SU-IgG to dunni cells by flow cytometry (Figure 4B). To address the binding specificity of this reagent, and by extension of our cloned SU sequence, we also examined the binding to dunni cells infected with replication competent $1 \mathrm{E}$ or with $4070 \mathrm{~A}$ amphotropic viruses. Similar binding of 1E-SU-IgG was observed in both control and dunni/4070A, whereas reduced binding was observed in dunni/1E cells. As a control, we found that Ampho-SUIgG protein binding to dunni cells was inhibited in cells infected by an amphotropic virus (Figure 4C). The ability of replication competent $1 \mathrm{E}$ virus to inhibit binding of $1 \mathrm{E}-$ SU-IgG to cells demonstrates that the cloned SU recognizes a protein that is also bound by the $1 \mathrm{E}$ virus isolate. From this result, we conclude that the cloned SU sequence is representative of the Env present in the $1 \mathrm{E}$ virus.

\section{Analysis of xenotropic and polytropic Env binding to cells expressing human, hamster and chimeric receptors}

The ability of AKR6-pseudotype vector to utilize chimeric receptors that contain either of two non-overlapping regions of hXpr1 suggests that this virus can bind independently to either of the two regions of the cellular receptor. To test this prediction, we measured binding of AKR6 virus to $\mathrm{CHO}$ cells expressing various receptors by FACS analysis (Figure 5) using a rat antibody (83A25) that recognizes epitopes in the C-terminus of Env but does not interfere with virus binding to cells [25]. We found a clear increase in AKR6 virus binding to cells expressing hXpr1 in comparison to cells expressing haXpr1. AKR6 virus binding to cells expressing the AAAU chimeric receptor 

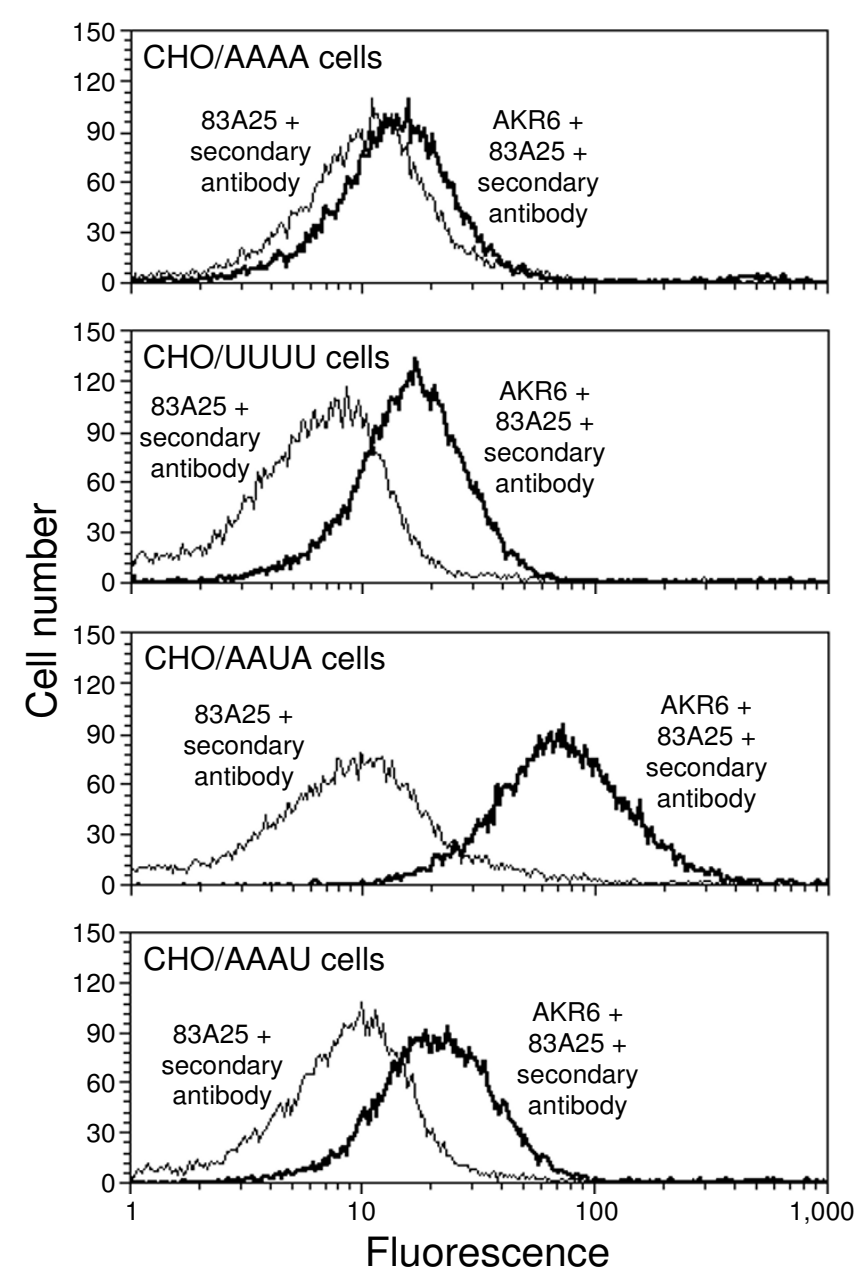

Figure 5

Measurement of AKR6 virus binding to cells expressing chimeric receptors. $\mathrm{CHO}$ cells transduced with retroviral vectors expressing hamster, human or chimeric $\mathrm{Xprl}$ receptor proteins were incubated with or without LAPSN(AKR6) virus and virus binding was detected by flow cytometry using the $83 \mathrm{~A} 25$ anti-Env primary and a fluorescent secondary antibody. Each histogram represents 14,000 to 18,000 live cells (cells that exclude propidium iodide). The experiments were repeated twice with similar results.

was similar to that of cells expressing hXpr1, consistent with the ability of the AAAU chimera to mediate entry of vectors bearing the AKR6 Env. Interestingly, AKR6 virus binding to cells expressing the AAUA chimera was much higher than that of cells expressing hXpr1. It is important to note that we have not determined the relative cell surface expression levels of the receptors and receptor chimera, and it is possible that differences in binding reflect varied protein levels as opposed to differences in binding affinities. However, binding of the AKR6 virus to cells expressing the AAUA and AAAU chimeras at levels at least as high as to cells expressing hXpr1 is consistent with the hypothesis that the AKR6 Env can independently bind the third or the fourth ECL of hXpr1.

The 1E-pseudotype vector could only utilize chimeric receptors that contained the fourth ECL of hXpr1, suggesting that only chimeric receptors containing the fourth ECL of hXpr1 would bind the $1 \mathrm{E}$ Env. In this case we could not measure $1 \mathrm{E}$ virus binding to cells because the $83 \mathrm{~A} 25$ rat antibody did not bind to the $1 \mathrm{E}$ Env (data not shown), in agreement with previous data showing that $83 \mathrm{~A} 25$ does not recognize Env from some strains of FrMCF [25]. Instead, to measure $1 \mathrm{E}$ Env binding we measured binding of the 1E-SU-IgG protein to cells expressing the chimeric receptors (Figure 6). 1E-SU-IgG binding to hXpr1 was higher than that to haXpr1, consistent with the difference in receptor activities of these proteins. 1E-SU-IgG binding to cells expressing the AAUA chimeric receptor was similar to that for cells expressing hXpr1 while binding to cells expressing the AAAU chimera was higher than that to AAUA- or hXpr1-expressing cells. These results indicate that the $1 \mathrm{E}$ Env can bind most efficiently to a receptor containing the fourth ECL (AAAU), but equal binding of $1 \mathrm{E}$ Env to AAUA and human Xpr1 was not expected based on the $1 \mathrm{E}$ vector transduction data. As with the AKR6 virus binding studies above, it is possible that differences in receptor expression may have influenced these results. In addition, there is relatively high binding of $1 \mathrm{E}-\mathrm{SU}$-IgG to haXpr1, a poor receptor for 1E-pseudotype vectors.

\section{Discussion}

Results obtained here with the hamster/human receptor chimeras are consistent with previous studies demonstrating the importance of residues within the putative third and fourth ECL of Mus dunni Xpr1 in xenotropic receptor function [21]. In that study, mutations in both the third and fourth ECL of Mus dunni Xpr1 were required to abolish xenotropic receptor function while mutations in either ECL alone did not limit virus entry. In the current study, the ability of AKR6 pseudotyped vectors to utilize either the AAUA or the AAAU chimera as a receptor demonstrates that either the third or fourth human ECL is sufficient to support X-MLV entry.

Taken together, our experiments with chimeric receptors suggest a model for entry of X-MLV and P-MLV that is consistent with the NRI observed previously, given that no XMLV specific receptor has been identified. We propose that two receptor functions are present simultaneously in different domains of Xpr1. One domain, which resides in the fourth ECL functions as a recognition site for both xenotropic and polytropic viruses, while the second receptor domain in the third ECL can only interact efficiently with xenotropic Env. 


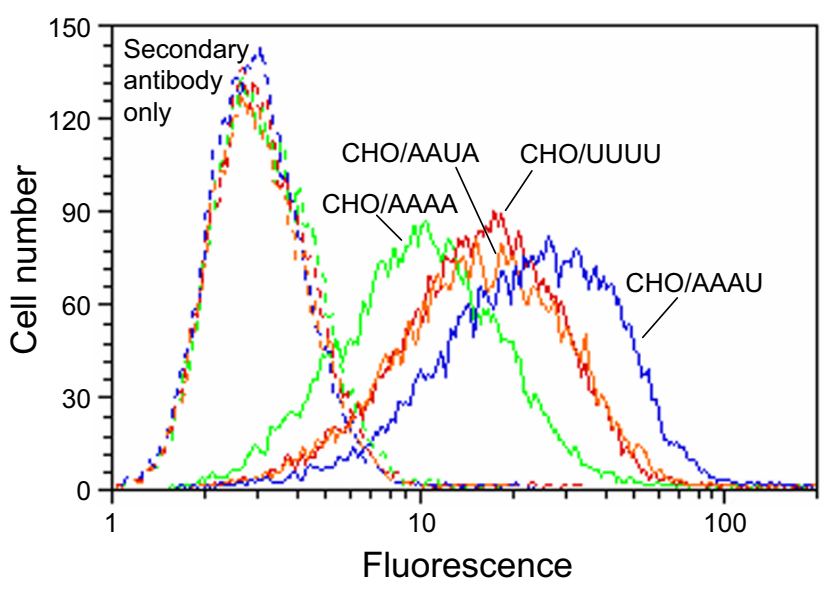

Figure 6

Measurement of IE-SU-IgG binding to cells expressing chimeric receptors. $\mathrm{CHO}$ cells transduced with retroviral vectors expressing hamster (AAAA, green), human (UUUU, red) or chimeric (AAUA, orange; AAAU, blue) XprI receptor proteins were incubated with (solid lines) or without (dashed lines) purified IE-SU-IgG, with fluorescent anti-lgG secondary antibody, and were analyzed by flow cytometry. All analyses were performed in the same experiment with the same FACS settings. Each histogram represents $\sim 13,000$ live cells (cells that exclude propidium iodide). The experiment was repeated once with similar results.

Our model for NRI predicts that the xenotropic and polytropic viruses should show a reciprocal pattern of interference in a receptor lacking the X-MLV specific receptor domain. The interference experiments described here using the AAAU and AAUU chimeras confirm this prediction. The interference pattern on the AAUU chimera, which contains both entry domains, is non-reciprocal due to the presence of the third extracellular loop. If the xenotropic specific determinant is removed, as in the AAAU chimera, X-MLV entry is markedly inhibited in cells expressing the $1 \mathrm{E}$ Env. This finding demonstrates that the third ECL is required for NRI, and that a chimeric receptor lacking this region serves as a common receptor for both P-MLV and X-MLV.

In the interference experiments described here, $1 \mathrm{E}$ Env was sometimes unable to completely block infection by a $1 \mathrm{E}$-pseudotype challenge vector (Table 2). Previous work suggests that such incomplete interference may reflect an inherent inability of P-MLV to completely block their cellular receptor. In vitro studies specifically examining the mechanism of P-MLV pathogenesis have shown that infection of cells by polytropic/MCF viruses results in accumulation of unintegrated extrachromosomal viral DNA, suggesting that P-MLV are capable of superinfecting cells in culture [26]. This finding is consistent with studies from other oncoretroviral systems showing that patho- genic viral stains can often superinfect cells [27-29]. Given that receptor mediated interference is the primary mechanism by which viruses prevent superinfection, the demonstrated ability of P-MLV to initiate multiple rounds of infection suggests that some polytropic Env proteins are inherently incapable of blocking certain receptors. However, it should be noted that strong interference by polytropic Env proteins can be observed in some cases (Table 2) $[4]$.

It is tempting to speculate that the regions we have identified through our chimera analyses represent the motifs within Xpr1 that are responsible for binding to the viral Env. The critical portions of the molecule are believed to lie outside of the cell, and therefore represent candidates for SU binding domains. However, it is difficult to accurately predict the topology of transmembrane receptors, as was shown in the case of Pit1 and Pit2. Initial predictions of receptor topology were used to design a number of chimeras similar to those described here. Regions within those chimeras were identified that enhanced infection by GALV or amphotropic MLV respectively, and it was suggested that these regions were responsible for virus binding [30-33]. However, recent experiments have provided a new, experimentally verified topology for Pit2 [34], and several of the previously identified critical regions were found to lie on the inner surface of the cell membrane. Therefore, before a specific role can be firmly assigned to the third and fourth ECL of Xpr1, the topology of the protein must be established.

\section{Conclusion}

Results presented here indicate that the non-reciprocal interference between polytropic and xenotropic retroviruses can be explained by a common receptor domain in the putative fourth ECL of Xpr1 and a specific receptor domain for xenotropic virus in the third ECL of the same Xpr1 protein.

\section{Methods}

\section{Virus and cell line nomenclature}

Cell lines containing integrated retroviral vectors are indicated by the name of the cell line, followed by a slash, followed by the name of the integrated vector (e.g. dunni/ LAPSN, or CHO/LN). Retroviral vectors in the viral form are described by the vector name followed, in parentheses, by the name of the replication-competent virus or packaging cell line used to produce the vector [e.g. LAPSN(AKR6), LAPSN(PA317)]. Where packaging cell lines have been used, the Gag and Pol proteins are from Moloney murine leukemia virus.

\section{Cell culture}

Chinese hamster ovary (CHO) cells (CHO-K1, ATCC CCL $61)$ were grown in minimum essential medium-alpha $(\alpha-$ 
MEM) (Gibco) supplemented with 10\% fetal bovine serum (FBS) (Hyclone). All other cell lines were grown in Dulbecco's minimal essential medium (DMEM) (Gibco) supplemented with $10 \%$ FBS. CHO cells expressing chimeric receptors were generated by calcium phosphatemediated transfection of receptor expression constructs. One day post-transfection, cells were trypsinized and seeded at 1:10 dilution into medium containing G418 ( $750 \mu \mathrm{g}$ active compound per $\mathrm{ml}$ ) and were maintained in selection medium for 7 to 10 days. Surviving cells were pooled and utilized in subsequent transduction assays. Mus dunni tail fibroblasts (dunni cells), the generation of dunni/LN, dunni/LAPSN, and helper virus-infected derivatives have been described [4]. LGPS/LAPSN cells [35] are a clone of NIH 3T3 cells that express Moloney MLV Gag and Pol proteins and contain the retroviral vector LAPSN [6]. Retrovirus packaging cell lines used included PA317 [36], PD223 [37] and FlyRD [38]. All cells were grown in a $37^{\circ} \mathrm{C}$ incubator at $10 \% \mathrm{CO}_{2}$ and $100 \%$ relative humidity.

\section{Chimeric receptor plasmids and retroviral vectors}

Receptor chimeras are named to indicate the origin of the sequence in each putative extracellular loop, based on the receptor topology model provided in Figure 1. This model has been suggested in previous studies [21], and was confirmed for this study by using a number of topology prediction algorithms located on the ExPASy proteomics server [39]. For the human/hamster Xpr1 receptor chimeras (Figure 1), "A" indicates sequence from the Cricetulus griseus hamster receptor [GenBank:AF198106], while a " $U$ " is used for the human sequence derived from a HeLa cell cDNA library [GenBank:AF099082]. Chimeric Xpr1 proteins were constructed by exchanging restriction fragments as indicated in Figure 1 . The $2 \mathrm{~kb}$ DNA fragments containing the hXpr1 or haXpr1 coding regions were blunt ended with Klenow and was cloned into SmaI digested pBluescript II (Stratagene, La Jolla CA). Following the exchange of fragments required to generate chimeric receptors, all constructs were confirmed by sequencing using primers internal to the receptor sequence. Retroviral vectors expressing the chimeric receptors were made by insertion of $2 \mathrm{~kb}$ XhoI-BamHI fragments containing the receptor coding regions from pBluescript into the retroviral expression plasmid LXSN [40] after digestion of pLXSN with HpaI and BamHI. Additional retroviral vectors used here included LAPSN [6], which encodes AP and Neo, and LN [40], which encodes Neo.

\section{Viruses and infection assays}

The AKR6 xenotropic and 1E polytropic virus isolates were a kind gift from Bruce Chesebro [14]. LAPSN(AKR6) and LAPSN(1E) retroviral vectors were generated by infecting dunni/LAPSN cells with AKR6 or 1E helper virus, as described previously [4]. LAPSN(AKR6env) and LAPSN(1Eenv) vectors were generated by transfection of pSX2-AKR6env and pSX2-1Eenv into LGPS/LAPSN cells using standard calcium phosphate protocols. Briefly, LGPS/LAPSN cells were plated into 6 -cm-diameter culture dishes at $5 \times 10^{5}$ cells per dish approximately $16 \mathrm{~h}$ prior to transfection. The following day, $9 \mu \mathrm{g}$ of the Env expression plasmid was transfected into the cells with $1 \mu \mathrm{g}$ of pCMV$\beta$ gal as a control for transfection efficiency. The following day cells were rinsed with PBS, and incubated with $4 \mathrm{ml}$ culture medium per plate overnight. The conditioned medium was collected, filtered through a $0.45 \mu \mathrm{m}$ poresize filter, and was frozen at $-80^{\circ} \mathrm{C}$. Vector titers were determined by limiting dilution assay on dunni cells. Additional viral vectors, including LAPSN (PA317), LAPSN (PD223), and LAPSN(FlyRD), were obtained by collecting conditioned medium from established producer lines.

Transduction assays in cell lines expressing chimeric receptors were carried out as follows. Approximately $16 \mathrm{~h}$ before infection, cell lines were plated at $7 \times 10^{4}$ cells/well into 6 -well $(\mathrm{d}=3.4 \mathrm{~cm})$ tissue culture dishes. Immediately prior to infection, medium was changed to include $4 \mu \mathrm{g} /$ ml Polybrene. Virus was added at appropriate dilutions, and the cells incubated for $48 \mathrm{~h}$ to allow expression of the alkaline phosphatase protein from the integrated LAPSN vector. Cells were then fixed in $3.7 \%$ formaldehyde in phosphate-buffered saline for $8 \mathrm{~min}$ at room temperature. Fixed cells were washed three times with phosphate-buffered saline. Endogenous alkaline phosphatase was inactivated by incubating the cells at $68^{\circ} \mathrm{C}$ for $1 \mathrm{~h}$. Cells were then stained for alkaline phosphatase activity by incubating the cells over night in AP staining buffer $(100 \mathrm{mM}$ Tris $\mathrm{pH} 8.5,100 \mathrm{mM} \mathrm{NaCl}, 50 \mathrm{mM} \mathrm{MgCl}{ }_{2}, 1 \mathrm{mg} / \mathrm{ml}$ Nitro Blue tetrazolium, $100 \mu \mathrm{g} / \mathrm{ml}$ X-Phos). Transduction events were measured by counting $\mathrm{AP}^{+}$foci.

\section{Env cloning}

Env SU sequences from the AKR6 [GenBank:DQ199948] and 1E [GenBank:DQ199949] viruses were obtained by PCR from low molecular weight DNA obtained from infected cells. Specifically, dunni cells were plated at $10^{5}$ cells in 6-cm-diameter tissue culture dishes. Following overnight incubation, the cells were infected at high multiplicity of infection $(\sim 100)$ with helper virus-containing stocks of LAPSN(AKR6) and LAPSN(1E) in the presence of $4 \mu \mathrm{g} / \mathrm{ml}$ Polybrene (Sigma). $16 \mathrm{~h}$ post-infection, low molecular weight DNA was isolated using the method of Hirt [41]. Env sequences corresponding to the SU portion of Env were isolated by PCR using primers Xeno5'env (5'ATGGAAGGTTCAGCGTTCTCAAAACCCC-3') and Xeno3'Env (5'-TGCCGCCCATAGTAAGTCCTCC-3'). Following gel purification using a Qiaquick gel purification kit (Qiagen), fragments were cloned into pCR2.1 using a 
Topo-TA cloning strategy (Invitrogen, Carlsbad CA). Full length Env coding regions were generated by ligation of a SacI-XhoI fragments into pBS-TM, a pBluescript-based vector containing a C-terminal fragment from the NZB env gene [GenBank:K02730]. The pBS-TM plasmid was made by insertion of a SacI-NotI fragment from pCSI-ENZB [16] into pBluescript II. Expression plasmids were generated by subcloning of XhoI-NotI fragments into pCR3.1 (Invitrogen) to generate pCR3.1-AKR6env and pCR3.1-1Eenv. To improve expression in murine and $\mathrm{CHO}$ cells, a BamHIHincII fragment containing the human cytomegalovirus immediate early promoter was replaced with a BamHINhel fragment containing the Moloney MLV LTR promoter and enhancer from pSX2 [42], to generate pSX2AKRenv and pSX2-1Eenv. These plasmids were sequenced to confirm the presence of complete Env open reading frames.

The 1E-SU-IgG plasmid was generated by ligation of a SacI-XhoI fragment from pCR2.1-1E-Env into pCI-NSU?9hFc [16]. To confirm the identity and integrity of the resulting fusion protein, the construct was sequenced using primers internal to the $1 \mathrm{E}-\mathrm{SU}$.

\section{Virus and Env SU binding assays}

Production and purification of 1E-SU-IgG for binding assays was carried out as described for other similar proteins $[43,44]$. For flow cytometry assays, $10^{6}$ cells were incubated with $1-2 \mu \mathrm{g}$ of purified fusion protein in a final volume of $100 \mu \mathrm{l}$ for $2 \mathrm{~h}$. Following washing, cells were incubated with a fluorescent anti human-IgG secondary antibody (DAKO F0315) for $1 \mathrm{~h}$. Cell fluorescence was determined by flow cytometry on a FACSCalibur (BD Biosciences), and data was analyzed using CellQuest software.

For virus binding assays, $10^{6}$ cells expressing the indicated receptor chimeras were incubated with LAPSN(AKR6) virus at $4^{\circ} \mathrm{C}$ for two h. Cells were washed three times with phosphate-buffered saline containing 2\% FBS and were incubated with $1 \mathrm{ml}$ hybridoma supernatant containing $83 \mathrm{~A} 25$ antibody for $2 \mathrm{~h}$. Following two additional washes and incubation with a FITC-conjugated anti-Rat-IgG secondary antibody, cell fluorescence was determined by flow cytometry using a FACSCalibur.

\section{Interference assays}

To establish $\mathrm{CHO}$ cell lines expressing high levels of AKR6 and $1 \mathrm{E}$ Env, cells were maintained in conditioned medium from dunni/LN cells (mock), or dunni/LN cells productively infected with AKR6 or 1E helper viruses. Conditioned medium ( $\alpha$-MEM with $10 \%$ FBS) was collected, centrifuged at $1,000 \times \mathrm{g}$ for $10 \mathrm{~min}$ to remove cells and debris, and frozen at $-80^{\circ} \mathrm{C}$ for $24 \mathrm{~h}$. Prior to addition to $\mathrm{CHO}$ cells, a 1:1 mixture of dunni conditioned medium and fresh $\alpha$-MEM with 10\% FBS was supplemented with $4 \mu \mathrm{g} / \mathrm{ml}$ Polybrene to facilitate infection. The conditioned medium mix was added to cells every $24 \mathrm{~h}$. As CHO cultures reached confluence (approximately every 3 days) cells were removed from the culture dish with trypsin/ EDTA and split 1:10 into new 6-cm-diameter dishes. After 6 weeks, cells were trypsinized, counted on a hemacytometer and plated at $10^{5}$ cells/well in 6 well dishes. Cells were then transduced with LAPSN(AKR) or LAPSN(1E) viral vectors. The titer of each vector was determined by limiting dilution. The degree of interference can be determined by comparing the vector titer on mock infected cells to that obtained on cells infected with AKR6 or 1E viruses.

\section{Competing interests}

The author(s) declare that they have no competing interests.

\section{Authors' contributions}

NSVH helped design the study, carried out the experiments, analyzed the data, and drafted the manuscript. ADM helped design the study and write the manuscript.

\section{Acknowledgements}

This study was supported by grants HL5488I, DK47754, and HL36444 from the NIH.

\section{References}

I. Weissenhorn W, Dessen A, Calder LJ, Harrison SC, Skehel JJ, Wiley DC: Structural basis for membrane fusion by enveloped viruses. Mol Membr Biol I999, I 6(I):3-9.

2. Colman PM, Lawrence MC: The structural biology of type I viral membrane fusion. Nat Rev Mol Cell Biol 2003, 4(4):309-319.

3. Sommerfelt MA, Weiss RA: Receptor interference groups of $\mathbf{2 0}$ retroviruses plating on human cells. Virology 1990, I 76(I):58-69.

4. Miller AD, Wolgamot G: Murine retroviruses use at least six different receptors for entry into Mus dunni cells. J Virol 1997, 7 I(6): 453 I-4535.

5. Ott D, Friedrich R, Rein A: Sequence analysis of amphotropic and IOAI murine leukemia viruses: close relationship to mink cell focus-inducing viruses. J Virol 1990, 64(2):757-766.

6. Miller DG, Edwards RH, Miller AD: Cloning of the cellular receptor for amphotropic murine retroviruses reveals homology to that for gibbon ape leukemia virus. Proc Natl Acad Sci USA 1994, 9 I(I):78-82.

7. van Zeijl M, Johann SV, Closs E, Cunningham J, Eddy R, Shows TB, $O$ 'Hara $B$ : A human amphotropic retrovirus receptor is a second member of the gibbon ape leukemia virus receptor family. Proc Natl Acad Sci U S A 1994, 9 I (3): I I 68-I I 72.

8. Miller $D G$, Miller AD: A family of retroviruses that utilize related phosphate transporters for cell entry. J Virol 1994, 68(1 2):8270-8276.

9. O'Hara B, Johann SV, Klinger HP, Blair DG, Rubinson H, Dunn KJ, Sass P, Vitek SM, Robins T: Characterization of a human gene conferring sensitivity to infection by gibbon ape leukemia virus. Cell Growth Differ 1990, I(3): I 19- 127.

10. Takeuchi Y, Vile RG, Simpson G, O'Hara B, Collins MK, Weiss RA: Feline leukemia virus subgroup $B$ uses the same cell surface receptor as gibbon ape leukemia virus. J Virol 1992, 66(2): $1219-1222$.

II. Brojatsch J, Naughton J, Rolls MM, Zingler K, Young JA: CARI, a TNFR-related protein, is a cellular receptor for cytopathic avian leukosis-sarcoma viruses and mediates apoptosis. Cell 1996, 87(5):845-855. 
12. Adkins HB, Brojatsch J, Naughton J, Rolls MM, Pesola JM, Young JA: Identification of a cellular receptor for subgroup $E$ avian leukosis virus. Proc Natl Acad Sci U S A 1997, 94(21): I I6 I7-I I 622.

13. Adkins HB, Blacklow SC, Young JA: Two functionally distinct forms of a retroviral receptor explain the nonreciprocal receptor interference among subgroups $B, D$, and $E$ avian leukosis viruses. J Virol 200I, 75(8):3520-3526.

14. Chesebro B, Wehrly K: Different murine cell lines manifest unique patterns of interference to superinfection by murine leukemia viruses. Virology 1985, I4I(I): II9-I29.

15. Tailor CS, Nouri A, Lee CG, Kozak C, Kabat D: Cloning and characterization of a cell surface receptor for xenotropic and polytropic murine leukemia viruses. Proc Natl Acad Sci U S A 1999, 96(3):927-932.

16. Battini JL, Rasko JE, Miller AD: A human cell-surface receptor for xenotropic and polytropic murine leukemia viruses: possible role in $\mathbf{G}$ protein-coupled signal transduction. Proc Natl Acad Sci USA 1999, 96(4): I385-1390.

17. Yang YL, Guo L, Xu S, Holland CA, Kitamura T, Hunter K, Cunningham JM: Receptors for polytropic and xenotropic mouse leukaemia viruses encoded by a single gene at Rmcl. Nat Genet 1999, 2 I(2):216-219.

18. Kozak CA: Genetic mapping of a mouse chromosomal locus required for mink cell focus-forming virus replication. J Virol I 983, 48(I):300-303.

19. Kozak CA: Susceptibility of wild mouse cells to exogenous infection with xenotropic leukemia viruses: control by a single dominant locus on chromosome I. J Virol I985, 55(3):690-695.

20. Spain BH, Koo D, Ramakrishnan M, Dzudzor B, Colicelli J: Truncated forms of a novel yeast protein suppress the lethality of a $\mathbf{G}$ protein alpha subunit deficiency by interacting with the beta subunit. J Biol Chem I995, 270(43):25435-25444.

21. Marin M, Tailor CS, Nouri A, Kozak SL, Kabat D: Polymorphisms of the cell surface receptor control mouse susceptibilities to xenotropic and polytropic leukemia viruses. J Virol 1999, 73(I I):9362-9368.

22. Levy JA, Pincus T: Demonstration of Biological Activity of a Murine Leukemia Virus of New Zealand Black Mice. Science 1970, I 70:326-327.

23. Levy JA: Xenotropic viruses: murine leukemia viruses associated with NIH Swiss, NZB, and other mouse strains. Science | 973, I 82(I | 7): I| I5I-II53.

24. Hartley JW, Wolford NK, Old LJ, Rowe WP: A new class of murine leukemia virus associated with development of spontaneous lymphomas. Proc Natl Acad Sci U S A 1977, 74(2):789-792.

25. Evans LH, Morrison RP, Malik FG, Portis J, Britt WJ: A neutralizable epitope common to the envelope glycoproteins of ecotropic, polytropic, xenotropic, and amphotropic murine leukemia viruses. J Virol 1990, 64(1 2):6176-6183.

26. Yoshimura FK, Wang T, Nanua S: Mink cell focus-forming murine leukemia virus killing of mink cells involves apoptosis and superinfection. J Virol 200 I, 75( I 3):6007-60I5.

27. Reinhart TA, Ghosh AK, Hoover EA, Mullins J: Distinct superinfection interference properties yet similar receptor utilization by cytopathic and noncytopathic feline leukemia viruses. J Virol 1993, 67(9):5153-5162.

28. Chen IS, Temin HM: Establishment of infection by spleen necrosis virus: inhibition in stationary cells and the role of secondary infection. J Virol I982, 4I(I): I83-I9I.

29. Weller SK, Joy AE, Temin HM: Correlation between cell killing and massive second-round superinfection by members of some subgroups of avian leukosis virus. J Virol 1980, 33(I):494-506.

30. Johann SV, van Zeijl M, Cekleniak J, O'Hara B: Definition of a domain of GLVRI which is necessary for infection by gibbon ape leukemia virus and which is highly polymorphic between species. J Virol I993, 67( I I):6733-6736.

31. Pedersen L, Johann SV, van Zeijl M, Pedersen FS, O'Hara B: Chimeras of receptors for gibbon ape leukemia virus/feline leukemia virus $B$ and amphotropic murine leukemia virus reveal different modes of receptor recognition by retrovirus. J Virol 1995, 69(4):240I-2405.

32. Pedersen L, van Zeijl M, Johann SV, O'Hara B: Fungal phosphate transporter serves as a receptor backbone for gibbon ape leukemia virus. J Virol 1997, 7 I(10):7619-7622.
33. Lundorf MD, Pedersen FS, O'Hara B, Pedersen L: Amphotropic murine leukemia virus entry is determined by specific combinations of residues from receptor loops 2 and 4 . J Virol 1999 , 73(4):3169-3175.

34. Salaun C, Rodrigues P, Heard JM: Transmembrane topology of PiT-2, a phosphate transporter-retrovirus receptor. J Virol 200I, 75(I 2):5584-5592.

35. Miller AD, Garcia JV, von Suhr N, Lynch CM, Wilson C, Eiden MV: Construction and properties of retrovirus packaging cells based on gibbon ape leukemia virus. J Virol 1991, 65(5):2220-2224.

36. Miller $A D$, Buttimore $C$ : Redesign of retrovirus packaging cell lines to avoid recombination leading to helper virus production. Mol Cell Biol 1 986, 6(8):2895-2902.

37. Wolgamot G, Rasko JE, Miller AD: Retrovirus packaging cells expressing the Mus dunni endogenous virus envelope facilitate transduction of $\mathrm{CHO}$ and primary hematopoietic cells. J Virol 1998, 72(I 2): I0242-10245.

38. Cosset FL, Takeuchi Y, Battini JL, Weiss RA, Collins MK: High-titer packaging cells producing recombinant retroviruses resistant to human serum. J Virol 1995, 69( I 2):7430-7436.

39. The ExPASy proteomics server [http://us.expasy.org/]

40. Miller $A D$, Rosman $G$ ]: Improved retroviral vectors for gene transfer and expression. Biotechniques 1989, 7(9):980-990.

41. Hirt B: Selective extraction of polyoma DNA from infected mouse cell cultures. J Mol Biol I 967, 26(2):365-369.

42. Miller AD, Chen F: Retrovirus packaging cells based on IOAI murine leukemia virus for production of vectors that use multiple receptors for cell entry. J Virol 1996, 70(8):5564-557 I.

43. Kurre P, Kiem HP, Morris J, Heyward S, Battini JL, Miller AD: Efficient transduction by an amphotropic retrovirus vector is dependent on high-level expression of the cell surface virus receptor. J Virol 1999, 73( I):495-500.

44. Van Hoeven NS, Miller AD: Improved enzootic nasal tumor virus pseudotype packaging cell lines reveal virus entry requirements in addition to the primary receptor Hyal2. Virol 2005, 79(I):87-94.

45. Battini JL, Heard JM, Danos O: Receptor choice determinants in the envelope glycoproteins of amphotropic, xenotropic, and polytropic murine leukemia viruses. I Virol 1992, 66(3): $\mid 468-1475$. 